Cahiers
de la Recherche
sur les Droits

Cahiers de la recherche sur les droits fondamentaux

16 | 2018

Les partis politiques

\title{
La notion de parti politique en Chine
}

The Concept of a Political Party in China

\section{Xiaowei Sun}

\section{OpenEdition}

Journals

Édition électronique

URL : https://journals.openedition.org/crdf/309

DOI : $10.4000 /$ crdf.309

ISSN : 2264-1246

Éditeur

Presses universitaires de Caen

Édition imprimée

Date de publication : 16 novembre 2018

Pagination : 59-67

ISBN : 978-2-84133-901-3

ISSN : 1634-8842

Référence électronique

Xiaowei Sun, «La notion de parti politique en Chine», Cahiers de la recherche sur les droits

fondamentaux [En ligne], 16 | 2018, mis en ligne le 16 novembre 2019, consulté le 14 novembre 2022.

URL : http://journals.openedition.org/crdf/309; DOI : https://doi.org/10.4000/crdf.309 


\title{
La notion de parti politique en Chine
}

\author{
Xiaowei SUN \\ Maître de conférences en droit public à l'université Bourgogne Franche-Comté \\ Centre de recherches juridiques de l'université de Franche-Comté (CRJFC)
}

\section{Le statut des partis politiques en Chine}

A. L'institutionnalisation des partis politiques fondateurs de la RPC

B. La question de la création de nouveaux partis politiques

\section{Les rôles des partis politiques en Chine}

A. Les " partis démocratiques" dans la coopération multipartite et la consultation politique

B. Le PCC en tant que parti politique au pouvoir

La notion de parti politique est introduite en Chine de l'Occident à la fin du XIX ${ }^{\mathrm{e}}$ siècle. Toutefois, le phénomène partisan y a toujours existé, en tant que groupement de personnes partageant un ensemble d'opinions et cherchant à prendre le pouvoir afin de faire prévaloir leurs opinions.

Or, jusqu'à la révolution de $1911^{1}$, le mot «党» (Dang), utilisé pour désigner un tel phénomène, était souvent associé à la conspiration et à la scélératesse. En effet, selon le confucianisme, pensée classique prédominante en Chine jusqu'au début du XX' $\mathrm{XX}^{\mathrm{e}}$ le but de s'associer en groupes était de faire prévaloir des intérêts particuliers et factionnels et, a fortiori, au détriment de l'intérêt commun. Un tel comportement était considéré comme contraire à l'éthique politique et indigne d'un gentilhomme lettré. Confucius enseignait ainsi à ses adeptes: «Le gentilhomme est vertueux et désintéressé, il se fonde dans la masse, mais ne conspire pas à ses désirs ${ }^{2}$.

En outre, à l'époque impériale, les conflits factionnels ${ }^{3}$ constituaient l'une des causes les plus importantes de l'instabilité gouvernementale. C'est pourquoi les souverains impériaux ont toujours réprimé la formation des groupes au sein du gouvernement ${ }^{4}$ jusqu'à la veille de la révolution de 1911. À ce titre, il convient de préciser que cette interdiction a vécu une évolution à l'orée du $\mathrm{XX}^{\mathrm{e}}$ siècle, marquée par la création du premier parti politique chinois au sens moderne du terme.

En 1894, au cours de la première guerre sino-japonaise (1894-1895), contestant la capacité du gouvernement impérial de la dynastie Qing ${ }^{5}$, à l'initiative de Sun Yat-sen ${ }^{6}$, un groupe de ressortissants chinois, composé de commerçants,

1. Cette révolution (辛亥革命) vise à renverser le gouvernement impérial en vue d'établir un gouvernement républicain. Elle débute avec le soulèvement de Wuchang (武昌起义) du 10 octobre 1911, et se termine par l'abdication du dernier empereur de la dynastie Qing, Puyi, le 12 février 1912

2. 《君子矜而不争, 群而不党》(toutes les traductions sont les nôtres).

3. 党争 (Dangzheng). Il convient à ce titre de citer les trois conflits factionnels qui ont eu lieu successivement sous trois dynasties, Tang, Song et Ming: le conflit Niu- Li (牛李党争) de 808 à 846, le conflit conservateurs-réformateurs (新旧党争) de 1069 à 1094 et le conflit Donglin (东林党 争) de 1593 à 1623. Ces trois grands conflits ont tous directement ou indirectement contribué à l'effondrement d'une dynastie.

4. Il convient tout de même de relativiser cette interdiction de principe. En effet, un jeune empereur, malgré le caractère sacré et impérieux de son autorité, n'est pas toujours en mesure de s'imposer à l'égard des grands dignitaires influents. Il laisse parfois se former des factions au sein de son gouvernement en jouant le rôle d'arbitre. L'effectivité de sa suprématie repose in fine sur un jeu d'équilibre entre les forces.

5. La dernière dynastie en Chine, les Qing, d'origine mandchoue, a régné sur l'empire du Milieu de 1644 à 1912.

6. 孙文 (Sun Wen), père fondateur de la République de Chine, est souvent connu en Occident sous le nom Sun Yat-sen, prononciation cantonaise de l'un des ses surnoms. Il est plus connu en Chine sous son surnom Sun Zhongshan. 
industriels et intellectuels, a fondé la Société pour le redressement de la Chine ${ }^{7}$ à Honolulu. Cette organisation avait un programme politique précis illustré par le slogan suivant: «Expulser les Tartares, refonder la Terre du Milieu, et établir un gouvernement unifié ${ }^{8}$. Elle s'était surtout dotée d'un statut définissant son organisation et son fonctionnement. Cette organisation était en effet le prédécesseur du Kuomintang 9 , parti politique qui a fondé la République de 1912 et qui a gouverné la Chine jusqu'en 1949. Cependant, non seulement cette organisation politique n'a pas été créée sur le territoire chinois, mais encore, elle a été déclarée illégale par le gouvernement impérial dès sa fondation, ce jusqu'en 1911 où celui-ci a levé l'interdiction de la création des partis politiques. Pendant cette période, d'autres organisations politiques ont été successivement créées, dont le Parti de l'unité impériale ${ }^{10}$ en 1910. Celui-ci soutenait une réforme du gouvernement impérial en vue de la fondation d'une monarchie constitutionnelle. Il fut le premier parti politique reconnu par le gouvernement impérial et légalement enregistré.

Il résulte de cette évolution que, dorénavant, le mot 《党» (Dang) ne signifie plus seulement une faction au sein de la classe gouvernante, mais une organisation politique à l'instar des partis politiques occidentaux. La nouvelle signification se distingue de l'ancienne en ce qu'elle se rattache à une nouvelle notion de politique introduite de l'Occident. Le terme chinois complet est dorénavant 《政党》(Zhengdang), qui traduit littéralement le terme «parti politique».

À partir du XX ${ }^{\mathrm{e}}$ siècle, la classe des lettrés ne forme plus un corps homogène et cohérent destiné à assurer le gouvernement du pays. Les idées occidentales enchantent les lettrés chinois, mais en même temps affaiblissent leur place dans l'exercice du pouvoir qui relève de leur monopole depuis près de dix siècles ${ }^{11}$. La révolution de 1911 n'a pas été complète. Elle a échoué à construire un "gouvernement républicain unifié». Le pouvoir a basculé aux mains des militaires. Entre la révolution de 1911 et la fondation de la République de 1912, les forces militaires centrales et locales se sont mutuellement arrêtées et entravées, ce qui a laissé un espace pour le développement des partis politiques et, a fortiori, du système parlementaire. Une centaine de partis politiques ont été créés, dont une trentaine ont joué un rôle plus ou moins important au sein du Parlement de la République de 1912. Les intellectuels ont profité du désordre créé par la révolution pour se joindre à une nouvelle forme de vie politique.

Force est de constater que ces partis post-révolutionnaires étaient tous des "partis cadres" au sens de Maurice Duverger ${ }^{12}$, qui réunissaient des notables et élites, loin de la population et de la réalité politique, caractérisée par la menace d'une guerre civile due à la compétition des forces militaires centrales et locales. En 1914, le président de la République, l'un des seigneurs de guerre les plus puissants ayant concentré tous les pouvoirs à son profit, a dissout le Parlement. Il a ensuite instauré un régime présidentiel, puis une monarchie impériale ${ }^{13}$. Son abdication et sa mort ont entraîné un désordre total permettant aux seigneurs militaires locaux de former de véritables royaumes au sein de la jeune république. Ainsi, les partis politiques se sont éclipsés avec l'effondrement de l'éphémère système parlementaire de la République de 1912.

Seul le Kuomintang s'est renforcé à travers des réformes inspirées du modèle bolchevique. L'influence bolchevique ne relevait pas de l'ordre idéologique, mais du domaine structurel. Sun Yat-sen a introduit le centralisme dans l'organisation du parti ${ }^{14}$, en rapprochant celui-ci des différentes classes sociales ${ }^{15}$. Grace à ces réformes, le Kuomintang a affermi son rôle de direction de l'État. Il est devenu le Parti d'État.

Le Parti communiste de Chine (ci-après PCC) a été créé pendant cette même période de réformes du Kuomintang, également inspiré du modèle bolchevique cette fois tant au niveau organisationnel qu'au niveau idéologique. Depuis sa création en 1921, le PCC a été, parmi d'autres partis politiques, un parti d'opposition, malgré deux périodes de coopération avec le Kuomintang ${ }^{16}$. Néanmoins, le PCC n'était pas un parti d'opposition comme les autres. Il disposait d'une force armée importante et d'environ un tiers du territoire national à la fin de la deuxième guerre sino-japonaise (1945). Il constituait de facto un État à part ${ }^{17}$.

7. 兴中会 (Xingzhonghui).

8. 《驱逐鞑虏，恢复中华，创立合众政府》

9. 国民党, Guomindang selon la version de la République populaire de Chine, littéralement traduit par Parti nationaliste.

10. 帝国统一党

11. En Chine, les lettrés ont toujours joué un rôle prééminent dans l'exercice du pouvoir. À partir du Ir siècle av. J.-C., ils commencent à représenter le personnel de l'administration impériale. Cette bureaucratie des lettrés s'est progressivement renforcée notamment à travers l'instauration du concours impérial à l'aube du VII ${ }^{\mathrm{e}}$ siècle. À partir du XI siècle, le concours impérial devient la seule voie d'accès aux dignités et offices impériaux. Le pouvoir est dorénavant entièrement aux mains des lettrés jusqu'au début du XX $\mathrm{XX}^{\mathrm{e}}$ siècle.

12. M. Duverger, Les partis politiques, $10^{\mathrm{e}}$ éd., Paris, Seuil (Points. Essais), 1992, p. 120-122.

13. Le 12 décembre 1915, soutenu par des monarchistes, le président de la République Yuan Shikai (袁世凯) se proclama empereur de l'Empire chinois (中华帝国). Mais cette restauration impériale ne dura que quatre-vingt-trois jours.

14. Une réforme a été effectuée en 1914 en vue de la centralisation du pouvoir au profit de Sun Yat-sen. Les membres du parti étaient hiérarchisés en fonction de leur date d'adhésion.

15. En 1919, Sun Yat-sen a décidé d'élargir le Parti nationaliste en intégrant notamment des membres issus de la classe non possédante. Il a conclu également des alliances avec l'URSS et le jeune Parti communiste de Chine soutenu par celle-ci.

16. La première période eut lieu de 1923 à 1927 en vue de l'unification de la République contre les seigneurs de guerre, puis la deuxième, de 1937 à 1946, forma une alliance contre l'invasion japonaise.

17. En 1945, autour de la reddition des forces japonaises, environ cent millions d'habitants étaient sous le gouvernement du PCC. Celui-ci contrôlait la plupart du territoire du nord de la Chine et disposait de plus d'un million de soldats. 
Au lendemain de la libération de l'occupation japonaise, en 1946, une Conférence consultative politique ${ }^{18}$ a été organisée en vue de mettre fin aux conflits entre le Kuomintang et le PCC qui déchiraient le pays. Cette Conférence était une sorte d'Assemblée pré-constituante qui réunissait les partis politiques les plus importants - dont le Kuomintang et le PCC comme principaux acteurs - ainsi que des personnalités notables dans divers domaines. Pendant la Conférence, les débats ont porté sur des questions relatives à la nature du régime politique (régime parlementaire/ régime présidentiel), à la détermination de la politique générale, à l'organisation des forces armées (séparation de l'armée et des partis politiques), à la composition de l'Assemblée nationale et au projet de Constitution. Cependant, les accords issus de la Conférence de 1946 n'ont pas été mis en place. Une nouvelle guerre civile a éclaté en 1947 entre le Kuomintang et le PCC, laquelle s'est soldée par la victoire de ce dernier. Or, la Conférence consultative politique demeurait la forme légitime de l'acte pré-constituant. Victorieux, le PCC a organisé en 1949 une nouvelle Conférence. De celle-ci est né le système de partis politiques de la future République populaire de Chine (ci-après RPC).

Pour le PCC, l'expérience du Kuomintang de 1912 à 1947 a été un échec. Le Kuomintang, représentant la bourgeoisie et une majorité de la classe intellectuelle, demeurait en réalité minoritaire par rapport à la population du pays. Or, il ne s'agissait pas d'une simple question de représentativité ou de technique électorale. Les conditions de droit électoral n'étaient pas si élevées ${ }^{19}$, alors que les électeurs étaient peu intéressés ${ }^{20}$. Inédit en Chine, le vote ne pouvait pas y jouer le même rôle qu'en Occident où, depuis l'Antiquité, il a toujours été un moyen de décision ou de désignation plus ou moins important selon les lieux et les époques. Par ailleurs, les circonstances d'invasion étrangère, d'occupation du territoire et de guerre civile n'étaient pas propices au développement d'un régime parlementaire.

Il résulte de ces faits que, sous la RPC, le système de partis politiques n'est pas structuré autour des élections. La Conférence consultative politique de 1949 a joué un rôle déterminant dans l'évolution du système de partis politiques en Chine. Depuis la fondation de la RPC, se développe un "système de coopération multipartite et de consultation politique sous la direction du Parti communiste de Chine ${ }^{21}$. Ce système a pour effet de figer la configuration des forces politiques, et de définir les rapports entre les partis politiques. Depuis la fondation de la RPC, il existe officiellement neuf partis politiques dont le PCC en tant que parti au pouvoir, tandis que les autres jouent un rôle de conseil et de surveillance. La notion de parti politique est inévitablement modelée par le développement de ce système.

Les spécificités de la notion chinoise de parti politique se manifestent non seulement à travers le statut des partis politiques (I), mais surtout à travers le rôle que jouent les partis politiques au sein de l'État (II).

\section{Le statut des partis politiques en Chine}

Comme en droit français, aucun texte juridique ne définit le parti politique en droit chinois. Or, plusieurs critères juridiques permettent de constater une institutionnalisation des neuf partis fondateurs de la RPC (A). À l'égard du statut de ces partis existants, se pose également la question de la création de nouveaux partis politiques (B).

\section{A. L'institutionnalisation des partis politiques fondateurs de la RPC}

L'institutionnalisation des partis politiques constitue l'une des particularités du système de partis politiques chinois. Ici, il ne s'agit pas de discuter de la confusion de l'État et du parti politique, mais d'analyser le phénomène selon lequel l'État dote les partis politiques d'un statut institutionnel.

Il s'agit des neuf partis politiques fondateurs de la $\mathrm{RPC}$, à savoir: le PCC, le Comité révolutionnaire du Kuomintang ${ }^{22}$, la Ligue démocratique de Chine ${ }^{23}$, l'Association pour la construction démocratique de Chine ${ }^{24}$, l'Association pour le progrès démocratique de Chine ${ }^{25}$, le Parti démocratique des paysans et ouvriers ${ }^{26}$, le Parti pour l'intérêt public ${ }^{27}$, la Société du trois septembre ${ }^{28}$, la Ligue du gouvernement autonome et démocratique de Taiwan ${ }^{29}$. Le PCC est le parti au pouvoir, tandis que

18. 政治协商会议

19. Selon la loi électorale en vigueur à l'époque, tout citoyen de la République de Chine, âgé de vingt ans, justifiant d'une résidence stable et régulière sur le territoire de la République d'une durée minimale de six mois, et jouissant de ses droits civils, a le droit de vote.

20. Selon les statistiques publiées par le New York Times, lors des élections législatives de 1947, sur les cent soixante-cinq millions d'électeurs inscrits, seulement vingt millions de bulletins ont été récoltés, soit $12 \%$ de participation (New York Times, 24 novembre 1947, p. 12).

21. 中国共产党领导的多党合作和政治协商制度

22. 中国国民党革命委员会. Après la défaite du Kuomintang et son retrait à Taiwan, une partie minoritaire de ses membres ont fait scission, formant le Comité révolutionnaire. Celui-ci s'est ensuite rallié au PCC.

23. 中国民主同盟, deuxième parti politique en Chine après le PCC et fidèle allié de celui-ci depuis sa fondation en 1941. Ses membres proviennent principalement des milieux intellectuels, d'enseignement et de recherche.

24. 中国民主建国会. Ses membres sont principalement des entrepreneurs du secteur de la fabrication, de la finance, ou des industries commerciales, et proviennent parfois des milieux culturels et intellectuels.

25. 中国民主促进会. Ses membres sont principalement des journalistes, des enseignants et des personnalités du domaine culturel.

26. 中国农工民主党, un parti qui rassemble des intellectuels du secteur médical et pharmaceutique.

27. 中国农工民主党, un parti principalement composé des rapatriés volontaires des Chinois d'Outre-mer.

28. 九三学社. Ses membres sont des intellectuels du domaine scientifique.

29. 台湾民主自治同盟. Fondé par les membres de l'ancien Parti communiste de Taiwan, les membres actuels de ce parti sont des personnes d'origine taïwanaise résidant sur le territoire de la RPC. 
les huit autres partis sont qualifiés de "partis démocratiques ${ }^{30}$. Selon le vocable chinois, la notion de "parti démocratique» doit être comprise dans le sens où les "partis démocratiques" participent à la démocratie de négociation ${ }^{31}$ qui implique un dialogue entre le PCC et les autres partis politiques. Ce dialogue se déroule sur la base de la Conférence consultative politique. C'est d'ailleurs la Conférence de 1949, organisée par ces neuf partis, sous l'impulsion du PCC, qui a donné naissance à la RPC. Le PCC et les huit "partis démocratiques» ont ensuite exercé ensemble le pouvoir législatif sous la forme de la Conférence consultative politique jusqu’à l'adoption de la Constitution de 1954 par la première Assemblée populaire nationale (ci-après APN). Dès lors, ils participent à la représentation au sein de l'APN, tout en continuant la coopération et la consultation politique en dehors du corps législatif.

En effet, les sièges et comités locaux de ces neuf partis sont des établissements publics. Une telle qualification ne se rattache à aucun texte juridique express, mais résulte de la combinaison de plusieurs critères. Il s'agit d'abord d'un critère budgétaire. Selon les Principes généraux du droit civil ${ }^{32}$ du 12 avril 1986, ces établissements sont dotés de la personnalité morale. Ils disposent par conséquent des fonds et ressources propres pour leurs activités. Une partie de ces fonds et ressources proviennent des finances publiques, tandis que le reste est issu des contributions (dons et cotisations) des membres du parti. À ce titre, il convient de distinguer les établissements du PCC de ceux des autres partis. Les établissements du PCC sont principalement financés par le Trésor public, alors que ceux des "partis démocratiques» sont largement financés par les contributions de leurs membres. En deuxième lieu, le caractère public des établissements des partis relève aussi d'un critère fonctionnel. Dès le début de la RPC, les établissements du PCC ont toujours fonctionné comme des établissements administratifs. D’ailleurs, les textes réglementaires sont souvent contresignés par le Comité central du PCC. La question se pose quant aux établissements des "partis démocratiques». Le rôle de ces établissements se limite à une fonction de coordination avec le PCC tant au niveau national qu'au niveau local. Ils n'ont aucune fonction administrative au-delà de la gestion interne. Or, leur fonction de coordination est considérée comme l'un des mécanismes essentiels de la coopération multipartite. Leurs activités se distinguent des simples activités non lucratives, sociales et culturelles.
Elles participent des fonctions de l'État. D'où le troisième critère, qui tient à la nature publique des personnels des établissements des partis politiques. En effet, selon le décret d'application de la loi du 27 avril 2005 sur les agents publics ${ }^{33}$, les dirigeants des comités centraux et locaux et les employés des établissements des partis politiques sont des fonctionnaires au sens de ladite loi. Ce décret prend soin de préciser, dans son annexe $n^{\circ} 1$, que sont concernés les établissements du PCC (art. 4) et ceux des huit «partis démocratiques» susmentionnés (art. 10). Un tel élargissement du champ d'application de la loi sur les agents publics a suscité nombre de critiques contestant la confusion des agents de l'État et des employés des établissements des partis politiques. Or, ces critiques n'ont pas visé le vrai problème. Il n'est pas gênant qu'un membre d'un parti politique devienne fonctionnaire. C'est d'ailleurs une situation courante dans la plupart des démocraties occidentales. D’un côté, le fonctionnaire jouit de sa liberté d'opinion en adhérant à un parti politique, de l'autre, il est normal que le parti au pouvoir puisse influencer l'administration par la nomination des agents partisans de son camp ${ }^{34}$. Le vrai problème, s'il en était un, est la neutralisation des identités politiques par l'inclusion des employés des établissements des partis politiques dans la fonction publique. Travailler au sein de l'établissement d'un parti politique, c'est servir l'État. Dès lors, les neuf partis politiques fondateurs de la RPC, exerçant des activités à caractère public avec le financement public et le personnel public, constituent une catégorie particulière d'institutions de l'État.

L'institutionnalisation des partis politiques fondateurs de la RPC a pour effet de pérenniser leur existence au risque d'effacer leurs identités politiques. Ces neuf partis monopolisent la représentation de la société entière. Or, celle-ci ne cesse d'évoluer depuis la fondation de la RPC, et parfois de manière radicale ${ }^{35}$. Il convient de s'interroger sur la possibilité et l'opportunité de créer de nouveaux partis politiques.

\section{B. La question de la création de nouveaux partis politiques}

Force est de constater que, depuis la fondation de la RPC, aucun nouveau parti politique n'a été légalement reconnu par l'État. Il est pourtant possible de créer de nouveaux partis politiques sous certaines conditions.

30. 民主党派 (Minzhudangpai). Pour éviter toute confusion, le terme "parti démocratique» utilisé pour désigner les huit partis chinois susmentionnés est toujours entre guillemets.

31. 协商民主. La démocratie de négociation constitue l'un des principes démocratiques de la RPC. Contrairement à la démocratie représentative, elle ne repose pas sur les élections. L'un des mécanismes de démocratie de négociation est la Conférence consultative politique.

32. 民法通则. Le pouvoir réglementaire a été habilité, en 1986, à fixer les grands principes dans le domaine du droit civil, en attendant l'élaboration d'un Code civil. L'APN a adopté, en 2017, les dispositions générales du Code civil.

33. 《中华人民共和国公务员法》实施方案, Conseil des affaires d’État, Comité central du PCC, 9 avril 2006.

34. Le système dit du Spoils System, pratiqué aux États-Unis d'Amérique jusqu'à la réforme du Pendleton Civil Service Reform Act en 1883 , en est un exemple par excellence.

35. Nous pouvons notamment citer la révolution culturelle de 1966 à 1976 et la grande réforme économique à partir des années 1980 . La Constitution a vécu deux révisions totales en 1975 puis en 1982. 
L'article 35 de la Constitution consacre la liberté d'association ${ }^{36}$. Ce principe constitutionnel constitue le fondement de la liberté de création des partis politiques. Comme en droit français, il est possible de créer un parti politique sous forme d'association. Mais, contrairement à la situation française, le législateur chinois n'est pas intervenu pour définir les conditions relatives à la création des associations. Une telle action est encadrée par le pouvoir réglementaire. Ainsi, le règlement du 25 octobre 1998 relatif à la gestion des enregistrements des associations sociales ${ }^{37}$ donne une définition à celles-ci dans son article 2 :

Sont des associations sociales au sens du présent règlement, les organisations non lucratives, librement constituées des citoyens chinois, en vue de la réalisation des objectifs communs de leurs membres, fonctionnant conformément à leurs statuts propres.

Lorsque ses objectifs sont de nature politique, l'association serait un parti politique. Aux termes de l'article 3 dudit règlement, la création d'association est soumise à un contrôle administratif préalable. L'administration exerce un pouvoir d'agrément discrétionnaire en la matière. En outre, l'association doit être immatriculée conformément aux dispositions issues dudit règlement. Ce dernier détermine, dans son article 10, les conditions relatives à la création des associations. Aux termes de cet article, une «association sociale» doit:

1. avoir au moins 50 membres personnes physiques ou au moins 30 membres personnes morales; en cas de composition mixte, au moins 50 membres personnes physiques et morales confondues;

2. être dotée d'un nom régulier et d'un statut propre; 3. avoir un domicile fixe;

4. disposer du personnel dédié à la réalisation de ses activités;

5. disposer de fonds et ressources financières légaux pour la réalisation de ses activités, à savoir 100000 yuans pour les associations au niveau national, 30 ooo yuans pour les associations au niveau local;

6. avoir la capacité d'assumer indépendamment la responsabilité civile.

Ce même article précise enfin que la désignation du nom de l'association doit être conforme aux dispositions législatives et réglementaires, et ne peut être contraire à la morale sociale. Le nom de l'association doit être en adéquation avec le champ de ses activités et l'étendue géographique de ses membres et activités, et refléter fidèlement ses caractéristiques. Depuis la modification du 6 février 2016 dudit règlement, la désignation des noms des associations au niveau national est soumise à l'autorisation des établissements compétents de l'État, tandis que les associations au niveau local ne peuvent être dotées des qualificatifs «chinois», "national» ou «de Chine».

Il est donc virtuellement possible de créer de nouveaux partis politiques, sous la forme d'association sociale. Mais la nature d'un tel parti politique serait évidemment différente de celle des neuf partis politiques existants, qu'il s'agisse du PCC ou des huit "partis démocratiques». Le parti créé sur la base du règlement de 1998 est une association, mais ne constitue pas une institution de l'État. Il ne saurait concourir à l'exercice du pouvoir comme le font les partis existants. Il pourrait participer aux élections comme dans un système représentatif occidental. À ce titre, la loi électorale ${ }^{38}$ offre la possibilité aux partis politiques de recommander des candidats à l'élection des représentants du peuple ${ }^{39}$. Mais la liste des candidats est définitivement établie par les unités électorales ${ }^{40}$. Concrètement, les unités électorales fixent le nombre de candidats pouvant figurer dans la liste de chaque circonscription (le nombre de candidats étant supérieur à celui des futurs élus), puis examinent les candidatures, et arrêtent la liste des candidats qui sera soumise aux électeurs. Quant à la procédure de cette sélection, quelque décisive qu'elle soit, la loi électorale se contente de prévoir la «discussion ${ }^{41}$ et la "négociation " ${ }^{42}$ entre les membres des unités électorales, sans préciser comment ces unités électorales sont constituées, ni le nombre de membres composant une unité. Mais, dans la pratique, les unités électorales sont organisées sous la direction des autorités administratives dans les lieux de travail et quartiers d'habitation.

En Chine, l'élection n'a pas vocation à désigner ceux qui vont gouverner pour une période déterminée, et le vote n'est pas un arbitrage entre les intérêts différents. Il est impossible de comprendre la notion chinoise de parti politique avec la logique d'un système représentatif. En tout état de cause, aucun nouveau parti politique n'a été reconnu sur la base du règlement de 1998. La notion chinoise de parti politique se traduit davantage par les rôles respectifs que jouent les neuf partis fondateurs de la RPC dans le système de coopération multipartite et de consultation politique sous la direction du PCC.

\section{Les rôles des partis politiques en Chine}

La Constitution affirme dans son préambule «le système de coopération multipartite et de consultation politique sous la direction du Parti communiste de Chine». Ce principe définit les rôles respectifs des partis politiques. Les huit "partis démocratiques » jouent un rôle essentiel dans

36. Art. 35 de la Constitution du 4 décembre 1982: «中华人民共和国公民有言论、出版、集会、结社、游行、示威的自由». Ce même principe a été affirmé par les Constitutions de 1954 et de 1975.

37. 社会团体登记管理条例, modifié le 6 février 2016.

38. 选举法

39. Art. 29, al. 2 de la loi électorale.

40. Art. 31 de la loi électorale.

41. 讨论

42. 协商 
la coopération multipartite et la consultation politique (A) qui se déroulent sous la direction du PCC (B).

\section{A. Les "partis démocratiques" dans la coopération multipartite et la consultation politique}

Différent des systèmes multipartites ou bipartites de l'Occident, et du régime de parti unique de certains pays, le système de partis politiques en Chine se caractérise par la direction du PCC et la participation au pouvoir des "partis démocratiques» par le biais de la coopération et de la consultation. Au lieu de former une opposition, les «partis démocratiques» sont des alliés du PCC, et participent aux affaires politiques.

La coopération multipartite et la consultation politique sont réalisées à travers la Conférence consultative politique tant au niveau national qu'au niveau local. À l'échelon national, les "partis démocratiques» et le PCC, entre autres associations sociales et personnalités notables indépendantes, sont représentés au sein de la Conférence consultative politique du peuple chinois. Cependant, celle-ci ne constitue pas un organe de l'État au sens de la Constitution, même si elle avait exercé le pouvoir législatif et préparé le projet de Constitution entre 1949 et 1954. Quant à sa nature juridique, la Constitution se contente d'une description brève et symbolique dans son préambule: «la Conférence consultative politique du peuple chinois est une organisation de Front uni pour une représentation extensive ${ }^{43}$. Aucun texte constitutionnel ni législatif ne définit la nature juridique de cet établissement. La Conférence détermine son propre statut. Il convient ici de distinguer les établissements de la Conférence en tant qu'établissements publics, de la Conférence en tant qu'institution politique. Comme dit supra, ces établissements sont dotés de la personnalité morale au sens des Principes généraux du droit civil. Ils peuvent infliger des sanctions disciplinaires à leurs personnels qui relèvent de la fonction publique. En revanche, lorsque la Conférence agit en tant qu'institution politique, ses activités n'ont aucun effet juridique et, en conséquence, elles ne sont soumises à aucun contrôle juridique. Cette sorte d'immunité juridique lui laisse une grande liberté d'action. Mais les résolutions de la Conférence n'ont aucune valeur juridique ni de force obligatoire, contrairement à l'APN qui exerce la compétence législative.

Or, nul n'ignore l'influence que la Conférence exerce tant sur les décisions politiques que dans le domaine du contrôle des actions publiques. Il convient de préciser que ses membres sont exclusivement des personnalités notables dans les différents domaines sociaux et professionnels (chefs des grandes entreprises, intellectuels reconnus, célébrités, personnages publics illustres, scientifiques renommés) ${ }^{44}$. Les membres de la Conférence ne sont pas élus, mais nommés sur recommandation des partis politiques, des organismes sociaux et culturels et des établissements publics. Si l'APN est censée représenter fidèlement la société selon les critères sociaux, économiques, ethniques et professionnels, la Conférence serait une véritable assemblée des élites. La consultation politique se réalise à travers les expertises et conseils que ces élites fournissent au PCC. L'existence d'un tel organisme politique a eu pour conséquence de changer les rapports entre le parti au pouvoir et les autres partis; ils ne sont pas dans un rapport de rivalité, mais plutôt de cordialité. Ce qui explique, en partie, l'absence de campagne électorale. En effet, la loi électorale ne mentionne qu'une simple présentation des candidats et la rencontre de ceux-ci avec les électeurs.

Cela étant, les "partis démocratiques" ne sont pas de simples auxiliaires du PCC. Ils jouent un rôle de surveillant du parti au pouvoir et du gouvernement. Une telle surveillance ne se réalise pas par le biais du mécanisme de l'opposition parlementaire ou des critiques médiatiques. L'APN, c'est-à-dire le Parlement chinois, exerce un contrôle de l'activité du gouvernement comme la plupart des régimes parlementaires. Mais les «partis démocratiques », bien que représentés au sein de l'APN, n'ont pas plus d'importance que les autres corporations et communautés sociales. En Chine, les élections ne sont pas des batailles de partis politiques, de même que le Parlement ne représente pas l'arène de la lutte partisane. L'APN serait une miniature de la société chinoise entière, alors que la Conférence consultative politique permet la participation des «partis démocratiques » à l'exercice du pouvoir tout en évitant la lutte partisane.

La Conférence est également le lieu et l'outil de la surveillance qu'exercent les "partis démocratiques » sur les activités du PCC et de son gouvernement. Selon le statut de la Conférence consultative politique du peuple chinois, cette surveillance consiste à évaluer et critiquer «l'application des normes constitutionnelles, législatives et réglementaires, la mise en œuvre des politiques générales et les activités des établissements administratifs et de leurs personnels ${ }^{45}$. Le PCC a pris soin de préciser la mise en œuvre de cette fonction de surveillance des «partis démocratiques ${ }^{46}$. Ainsi, dans un règlement intérieur (à titre expérimental) du 18 mai 2015 relatif aux activités de Front uni du Parti communiste de Chine, le Comité

43. 《中国人民政治协商会议是有广泛代表性的统一战线组织》.

44. À titre d'information, le Dalaï-Lama avait présidé la Conférence avant sa rupture avec le gouvernement. Le dernier empereur a été membre de cette assemblée.

45. Art. 2 du statut de la Conférence consultative politique du peuple chinois: 《对国家宪法、法律和法规的实施，重大方针政策的贯彻执行、 国家机关及其工作人员的工作，通过建议和批评进行监督》。

46. En 1989, le Comité central du PCC a publié l'avis relatif au maintien et à l'amélioration du système de coopération multipartite et de consultation politique sous la direction du Parti communiste de Chine (中共中央关于坚持和完善中国共产党领导的多党合作和政治协商制度的意见) 
central du PCC énumère dix domaines dans lesquels les "partis démocratiques» exercent leur surveillance par le biais de suggestions, critiques et évaluations ${ }^{47}$. De manière plus générale, le Conseil des affaires d'État a publié, en 2007 , le Livre blanc sur «le système de partis politiques en Chine ${ }^{48}$, confirmant la fonction de surveillance des "partis démocratiques» dans les domaines fixés par le statut de la Conférence consultative politique, en y ajoutant la surveillance des activités des comités et dirigeants du PCC.

Or, quel que soit l'étendue du domaine de surveillance, la forme de celle-ci se limite aux suggestions, critiques et évaluations qui n'entraînent pas de conséquence juridique. Les propositions de «juridicisation" de cette fonction de surveillance sont nombreuses et se renouvellent périodiquement. Mais ces propositions sont souvent considérées comme inadaptées au contexte social et politique chinois qui exige le maintien d'un exécutif fort et efficace. De surcroît, le système actuel serait plus conforme à la tradition politique chinoise qui recherche l'harmonie au lieu de trancher le conflit.

In fine, la forme et le contenu de la surveillance qu'exercent les "partis démocratiques» dépendent de la volonté du PCC qui assure la direction de l'État en tant que parti au pouvoir.

\section{B. Le PCC en tant que parti politique au pouvoir}

Dans le contexte chinois, dire que le PCC est le parti politique au pouvoir implique un double sens. Le premier est le sens connu des régimes parlementaires occidentaux: le parti au pouvoir est le parti majoritaire au Parlement. Il convient de préciser qu'en Chine les députés ne sont pas présentés en fonction de leurs étiquettes partisanes. Seuls les critères de sexe, d'ethnie et d'attachement territorial sont pris en compte ${ }^{49}$. Or, si l'on se réfère à certains rapports de
l'APN analysant la composition de la nouvelle législature à l'issue des élections nationales, force est de constater que les membres du PCC occupent autour de $70 \%$ des sièges à l'APN ${ }^{50}$. Avec cette majorité absolue, constante et stable, le PCC peut faire voter toutes les lois qu'il souhaite mettre en place. L'APN serait l'un des outils de la réalisation de sa politique. Jusqu'ici, le fonctionnement est tout à fait comparable avec les systèmes parlementaires occidentaux. Or, la place dominante du PCC au sein de l'État ne se fonde pas seulement sur une majorité parlementaire. Il convient d'introduire le deuxième sens du parti au pouvoir concernant le PCC.

Selon ce deuxième sens, la direction des affaires publiques par le PCC est un fait confirmé par le droit. En premier lieu, victorieux de la guerre civile, le PCC a fondé la RPC avec l'adhésion des huit «partis démocratiques". Depuis, le PCC a toujours exercé le rôle de direction, en décidant la politique générale du pays, en en précisant les mesures d'application et, parfois, en les faisant exécuter directement par ses cadres. Cette confusion de l'État et du Parti a été entérinée par une révision totale de la Constitution en 1975. L'article 2 de la Constitution de 1975 reconnaissait explicitement le rôle directeur du PCC au sein de l'État ${ }^{51}$. Par ailleurs, l'article 15 de ce même texte soumettait directement les forces armées à la direction du PCC 52 .

Depuis la réforme des années 1980, le PCC a promu une politique de séparation du Parti et de l'État ${ }^{53}$, qui s'est concrétisée notamment par la construction de l'État de droit et celle d'une fonction publique de l'État. De surcroît, la révision totale de la Constitution en 1982 a supprimé les deux articles susmentionnés reconnaissant la direction du PCC au sein de l'État. Le PCC a disparu des articles de la Constitution. Pourtant, on le retrouve dans son préambule où le rôle majeur du PCC dans la fondation de la RPC et sa fonction de direction de l'État sont affirmés. En outre, l'article ${ }^{\text {er }}$, affirmant la place dominante de la

Ce texte a fixé le cadre de la mise en œuvre de la surveillance qu'exercent les «partis démocratiques». Un avis de 2005 relatif au renforcement du système de coopération multipartite et de consultation politique sous la direction du Parti communiste de Chine (中共中央关于进一步加强中 国共产党领导的多党合作和政治协商制度建设的意见) a précisé la nature, le contenu et les formes d'une telle surveillance. Dans le même sens, très récemment, le PCC a publié un avis du 31 mars 2017 relatif au renforcement et à l'amélioration des activités de surveillance de la Conférence consultative politique du peuple (关于加强和改进人民政协民主监督工作的意见)

47. 中国共产党统一战线工作条例 (试行), art. 14 .

48. 《中国的政党制度》白皮书, Conseil des affaires d'État, 15 novembre 2007.

49. Voir les statistiques issues du Bureau national des statistiques de la République populaire de Chine (中华人民共和国国家统计局): http://www. stats.gov.cn/ztjc/ztsj/hstjnj/sh20o8/201208/t20120828_73585.html.

50. Il s'agit des rapports relatifs à l'explication de l'examen de la validité des élus de la nouvelle législature (全国人民代表大会代表资格的审查报告). À l'occasion de chaque renouvellement de l'APN, le Comité permanent de l'APN actuel désigne une commission chargée d'examiner la validité des résultats des nouvelles élections. Cette commission présente un rapport relatif à cet examen et, le cas échéant, des notes d'explications. La mention du pourcentage des membres du PCC n'est pas systématique dans ces rapports. Nous avons recensé trois rapports mentionnant cet élément. Selon les rapports pour la VI ${ }^{\mathrm{e}}(1983)$, la VII ${ }^{\mathrm{e}}$ (1988) et la IX ${ }^{\mathrm{e}} \mathrm{APN}$ (1998), les pourcentages des membres du PCC à l'APN sont respectivement 62,5\%, $66,8 \%$ et $71,48 \%$

51. Art. 2, al. 1 de la Constitution de 1975: «Le Parti communiste de Chine est le noyau dirigeant des peuples de la Chine entière. La classe ouvrière exerce sa direction sur l'État par l'intermédiaire de son détachement d'avant-garde, le Parti communiste de Chine» (《中国共产党是全中国人 民的领导核心。工人阶级经过自己的先锋队中国共产党实现对国家的领导》).

52. Art. 15, al. 1 et 2 de la Constitution de 1975: «L'armée de la Libération du peuple chinois et les milices sont des forces armées sous la direction du Parti communiste de Chine [...]. Le président du Comité central du parti communiste de Chine dirige les forces armées de l’État» («中国人民 解放军和民兵是中国共产党领导的工农子弟兵 [...] 中国共产党中央委员会主席统率全国武装力量》).

53. Dans son rapport du 25 octobre 1987, le secrétaire général du PCC, Zhao Ziyang, expose, devant l'assemblée plénière du XIII ${ }^{e}$ congrès national du PCC, la politique de séparation du Parti et de l'État. 
classe ouvrière au sein de la République ${ }^{54}$, est conservé avec une légère modification ${ }^{55}$. Il convient de rappeler que le PCC monopolise la représentation de la classe ouvrière. Ainsi, une lecture combinée du préambule et de l'article $1^{\mathrm{er}}$ de la Constitution permet de confirmer la direction du PCC au sein de l'État.

En effet, la séparation du Parti et de l'État ne signifie pas le retrait du PCC des affaires étatiques. Il suffit de lire le rapport du 25 octobre 1987 prononcé par le secrétaire général, Zhao Ziyang, devant l'assemblée plénière du XIII ${ }^{\mathrm{e}}$ congrès national du PCC. Il en ressort que le but de la séparation du Parti et de l'État est de renforcer la direction du PCC. Pour ce faire, il est nécessaire de décharger le Parti des tâches d'exécution, et de concentrer ses activités sur l'orientation et la détermination de la politique générale et sur le contrôle de la mise œuvre de ses décisions ${ }^{56}$. Récemment, lors d'une réunion de la $\mathrm{XII}^{\mathrm{e}} \mathrm{APN}$, le député Wang Qishan, l'une des personnalités les plus influentes au sein du PCC ${ }^{57}$, a précisé que la séparation du Parti et de l'État n'est pas organique, mais fonctionnelle. Selon lui, les établissements du PCC doivent être incorporés dans le corpus de l'État, celui-ci étant considéré au sens large du terme dans le contexte chinois, c'est-à-dire l'ensemble des institutions exerçant le pouvoir politique. En effet, non seulement toutes les institutions de l'État (tant fonctionnelles que territoriales) sont supervisées par un comité du PCC, mais encore, le PCC est organisé par une structure strictement hiérarchisée laquelle structure double celle de l'État. Si, naguère, l'État était envahi par le Parti, dorénavant, c'est le Parti qui subit une sorte d'étatisation. La construction de l'État de droit, officiellement affichée par la révision constitutionnelle de $1999^{58}$, consolide cette évolution. Le PCC considère le droit comme un instrument de gouvernement légitime et efficace. La réalisation de sa politique s'effectue désormais par le biais de l'édiction des normes juridiques. À ce titre, la récente révision constitutionnelle proposée par le Comité central du PCC en est une illustration parfaite ${ }^{59}$.

Parmi les vingt-et-une modifications, trois aspects attirent particulièrement notre attention. Il convient, en premier lieu, de noter le retour symbolique de la mention du PCC dans les articles de la Constitution. L'article $1^{\text {er }}$ de la Constitution, définissant la nature socialiste de la RPC, est complété par la phrase suivante: «La direction du PCC est le caractère essentiel du socialisme à la chinoise». Néanmoins, nous ne sommes plus dans le contexte de révolution culturelle des années 1970 où les organes de l'État s'effacent au profit des comités révolutionnaires. Le PCC, après une phase révolutionnaire et celle du développement économique, entre dans une ère nouvelle marquée par le souci de légitimation de son pouvoir. Tant que la construction de l'État de droit contribue à cette légitimation, la direction de l'État par le PCC doit être logiquement affirmée par la norme fondamentale.

La deuxième série de modifications consiste à instaurer une Commission nationale de surveillance ${ }^{60}$ et des commissions de surveillance à l'échelon supra-cantonal ${ }^{61}$. La Commission nationale de surveillance est un organe de l'État qui dérive directement de l'APN au même rang que la présidence de la République, le Conseil des affaires d'État (le Gouvernement populaire central), la présidence de la Commission militaire centrale, la Cour populaire suprême et le Parquet populaire suprême. Une nouvelle section est insérée dans le chapitre III de la Constitution, définissant le statut de ces commissions ${ }^{62}$. Par ailleurs, la fonction de surveillance est retirée du gouvernement au niveau central comme au niveau local ${ }^{63}$. Les commissions de surveillance interviennent essentiellement en matière de discipline et de lutte contre la corruption, avec le concours des comités de discipline du PCC et des parquets populaires. À l'issue de leurs enquêtes, elles peuvent proposer, voire décider des sanctions administratives - compétence jusqu'alors attribuée au pouvoir exécutif-, et suggérer des sanctions pénales - compétence relevant normalement du Parquet populaire. Il s'agit de l'une des réformes les plus importantes depuis le mandat du président Xi Jinping. Afin de mener à terme ces réformes, considérées comme indispensables à la réalisation $\mathrm{du}$ «Grand renouveau de la nation chinoise ${ }^{64}$, le PCC a jugé nécessaire de pouvoir prolonger le mandat du chef de l'État actuel, principal inspirateur de ces réformes.

Tel est le troisième objet de la révision constitutionnelle: la suppression de la limitation du nombre de mandats présidentiels ${ }^{65}$. D'aucuns évoquent le «mandat à vie» du président Xi Jinping. À ce propos, il convient de noter que la suppression de la limitation du nombre de

54. Art. ${ }^{\text {er }}$ de la Constitution de 1982: «La République populaire de Chine est un État socialiste de dictature démocratique du Peuple, dirigé par la classe ouvrière et fondé sur l'alliance des ouvriers et des paysans»（«中华人民共和国是工人阶级领导的、以工农联盟为基础的人民民主专 政的社会主义国家》).

55. La formule «dictature du prolétariat» est remplacée par «dictature démocratique du Peuple».

56. Secrétaire général du PCC, rapport du 25 octobre 1987, XIII congrès national du PCC.

57. L'un des conseillers les plus importants de l'actuel secrétaire général Xi Jinping, Wang Qishan a été membre du Bureau politique du Comité central et secrétaire général du Comité central de discipline du PCC.

58. La révision constitutionnelle de 1999 a introduit le principe de l'État de droit dans l'article 5 de la Constitution.

59. Le 26 janvier 2018, le Comité central du PCC a déposé au Comité permanent de l'APN une proposition de révision constitutionnelle. Cette révision est adoptée, le 11 mars 2018, par l'assemblée plénière de la XIII ${ }^{\mathrm{e}} \mathrm{APN}$.

60. Art. 62, 63, 65 et 67 de la Constitution.

61. Art. 101,103 et 104 de la Constitution.

62. La nouvelle section VII est intitulée «Les commissions de surveillance», comprenant cinq articles.

63. Art. 89 et 107 de la Constitution.

64. Slogan de la politique réformatrice de Xi Jinping, le «Grand renouveau de la nation chinoise» sera mentionné à deux reprises dans le préambule de la Constitution d'après la révision proposée par le PCC.

65. Art. 79 de la Constitution. 
mandats n'implique pas le «mandat à vie», lequel pourrait être explicitement constitutionnalisé ${ }^{66}$. Le président de la République continue à être élu par l'APN, et la durée de son mandat correspond à celle d'une législature. Même s'il est largement prévisible que Xi Jinping serait réélu pour un troisième, voire un quatrième mandat ${ }^{67}$ - tel est le but de cette révision -, sa réélection n'est pas systématique, encore faut-il qu'il soit candidat. Xi Jinping sera réélu à condition qu'il soit toujours indispensable à la direction du PCC et que cette direction soit favorable au développement du pays. Le PCC a parfois besoin d'un homme de la situation, comme Mao Zedong pendant la guerre civile ou Deng Xiaoping au lendemain de la révolution culturelle; mais il ne se laisserait pas être réduit à un instrument de domination personnelle.

La révision constitutionnelle de 2018 inscrit, par ailleurs, la pensée de Xi Jinping dans la lignée des pensées marxiste-léniniste, de Mao Zedong et de Deng Xiaoping, énoncées dans le préambule de la Constitution. En effet, le premier mandat de Xi Jinping a démontré son efficacité pour l'amélioration générale du niveau de vie des citoyens malgré des crises économiques latentes, l'apaisement des tensions sociales dues à des inégalités de jour en jour accentuées, et la lutte contre la corruption. Selon la tradition politique chinoise, ces faits seraient seuls suffisants pour justifier la continuation du gouvernement du PCC sous la direction de Xi Jingping. Or, le PCC considère que cette justification doit être traduite par le droit. Il est de fait que le PCC maîtrise le pouvoir normatif, y compris le pouvoir constituant. Or, il s'engage également à respecter les normes qu'il a mises en place. De surcroît, l'édiction des normes internes du PCC remplit les mêmes exigences relatives à la production des normes juridiques. Cette perspective se rapproche du concept d'État de droit, même si celui-ci doit être compris dans un sens purement formel ${ }^{68}$

La construction de l'État de droit, l'institutionnalisation de ses établissements et la neutralisation de son identité politique convergent vers une transformation du PCC. Celui-ci ne saurait être qualifié de simple parti politique au pouvoir. La confusion du Parti et de l'État est relayée par leur fusion. Si la RPC était un État de droit (même au sens formel), le PCC serait, si nous nous autorisons un néologisme, un «Parti de droit».

66. C'est le cas par exemple du Sénatus-consulte du 14 thermidor an X (2 août 1802), qui proclame Napoléon Bonaparte consul à vie. Il en est de même pour l'article 8 de la Constitution du 17 février 1807 d'Haïti qui dispose que «la charge de président et de généralissime des forces de terre et de mer est à vie».

67. Il n'aura raisonnablement que 80 ans au terme de son quatrième mandat présidentiel en 2032 . Selon la tradition du PCC, le successeur du chef de l'État actuel est souvent connu à la fin du premier mandat de celui-ci, et sera désigné comme vice-président de la République pour la prochaine législature. Il s'agit en principe d'un membre du Bureau politique du Comité central du PCC, âgé de moins de 65 ans. Pour le moment, parmi les favoris à la nomination du vice-président, personne ne remplit ces deux conditions coutumières.

68. Lorsque l'on évoque l'État de droit en Occident, celui-ci contient des éléments de démocratie libérale, tels que la séparation des pouvoirs, l'indépendance du pouvoir judiciaire, la reconnaissance et la protection des droits et libertés fondamentaux. L'état actuel de la construction de l'État de droit en Chine ne satisfait pas encore ces exigences substantielles. Si la reconnaissance et la protection des droits et libertés progressent de manière considérable, un obstacle idéologique demeure pour qu'un système de séparation des pouvoirs soit installé. En effet, le système de la RPC est fondé sur le principe de l'unité du pouvoir, laquelle unité est traduite par la souveraineté de l'APN. Tel est l'esprit de la Constitution : tous les organes de l'État découlent de l'APN. Une distinction fonctionnelle des pouvoirs s'effectue entre les organes dérivés sous la surveillance de l'APN. Selon cette logique, le pouvoir judiciaire ne saurait être indépendant. Il est soumis au pouvoir législatif. 